\title{
Laser Photoacoustic Spectroscopy
}

\author{
Markus W. Sigrist, Zurich
}

(ETH, Institute of Quantum Electronics, $\mathrm{CH}-8093$ Zurich)

Photoacoustic spectroscopy (PAS), also known as optoacoustic spectroscopy, was pioneered by Alexander Graham Bell more than a century ago. The advent of the laser strongly stimulated research and applications of this technique and the interest is such that international topical meetings on photoacoustic and photothermal phenomena are now held biennially.

The photoacoustic (PA) effect is essentially an energy-conversion process. When a sample (solid, liquid or gas) is irradiated by a laser beam or some other radiation source, part of the absorbed energy is converted into translation energy of the molecules by radiationless transitions. It is this de-excitation channel which is responsible for heat production within the sample although secondary reactions may also play a role (see Fig. 1). If either the incident radiation or the absorption by the sample is modulated, the periodic heating finally results in a pressure modulation. The PA signal thus originates from the thermal response of the sample to the absorbed energy detected as pressure fluctuations acoustically. Owing to thermal diffusion processes the magnitude of this signal depends not only on the thermal and optical characteristics of the sample but also on the nature of the coupling mechanism between sample and detector. Consequently the coupling medium plays an important role and one can differentiate between the light-to-heat conversion and the heatto-sound conversion efficiencies.

\section{Experimental Arrangement}

Since the time of Bell who used the Sun as radiation source, a foot-operated chopper for modulation and an earphone as acoustic detector, numerous experimental arrangements have been developed which mainly differ in the radiation sources employed and the system of both modulation and detection. Since the PA signal amplitude is proportional to the radiation power, the high spectral brightness offered by lasers is advantageous for the measurement of small absorptions, i.e. for weakly absorbing media or trace detection.

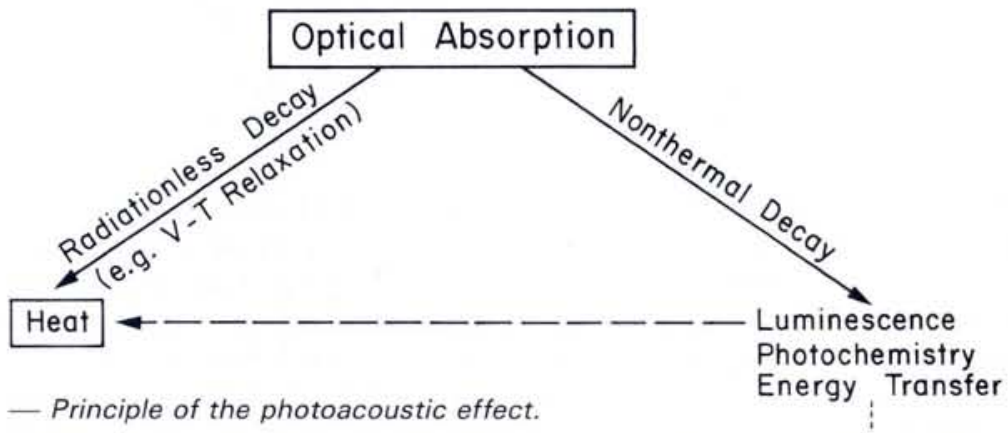

Two types of modulation should be distinguished: modulation of the incident radiation and modulation of the sample absorption. Schemes for the first include the widely-used amplitude modulation by mechanical choppers, electro-optic and acousto-optic modulators and the direct modulation of the radiation source including notably pulsed sources as well as frequencyor wavelength-modulation. These last offer the advantage of eliminating the continuum background PA signal resulting from a wavelength-independent absorption, e.g. of the entrance window of the PA cell containing the sample. On the other hand, modulation of the $a b$ sorption characteristics of the sample can be achieved on the basis of the Zeeman or Stark effect, by applying modulated magnetic or electric fields to the sample. Thereby the absorption wavelength is varied corresponding to a wavelength modulation and the continuum background is again suppressed.

While the design of the PA cell is not critical for solid or liquid samples, it may represent a crucial point for studies on

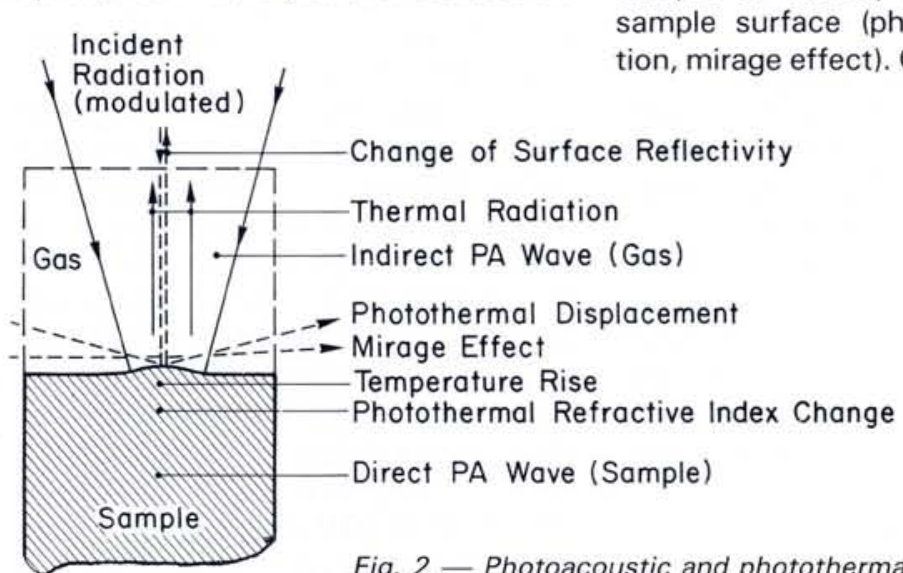

weakly absorbing gases. In these cases acoustically resonant cells are often used. The modulation frequency is then tuned to a resonance frequency of the cell preferably with pressure nodes at the cell windows to reduce the background originating from window absorption.

Finally, various different schemes exist for the detection of the thermal or acoustic disturbances caused by the absorbed radiation. The choice depends on the sample, the sensitivity to be achieved, ease of operation, ruggedness, and any requirement for non-contact detection, e.g. in aggressive media. The methods which have been developed for solid samples are summarized in Fig. 2; they include the measurement of the generated pressure wave either directly in the sample with a piezoelectric sensor for the pulsed regime, or indirectly in the gas above the sample with a microphone (photoacoustics). Induced changes of the refractive index can be sensed by monitoring the deflection of a probe beam, e.g. from a $\mathrm{HeNe}$ laser, either within the (transparent) sample or directly above the (plane) sample surface (photothermal deflection, mirage effect). Changes of the sur-

Fig. 2 - Photoacoustic and photothermal detection schemes. 


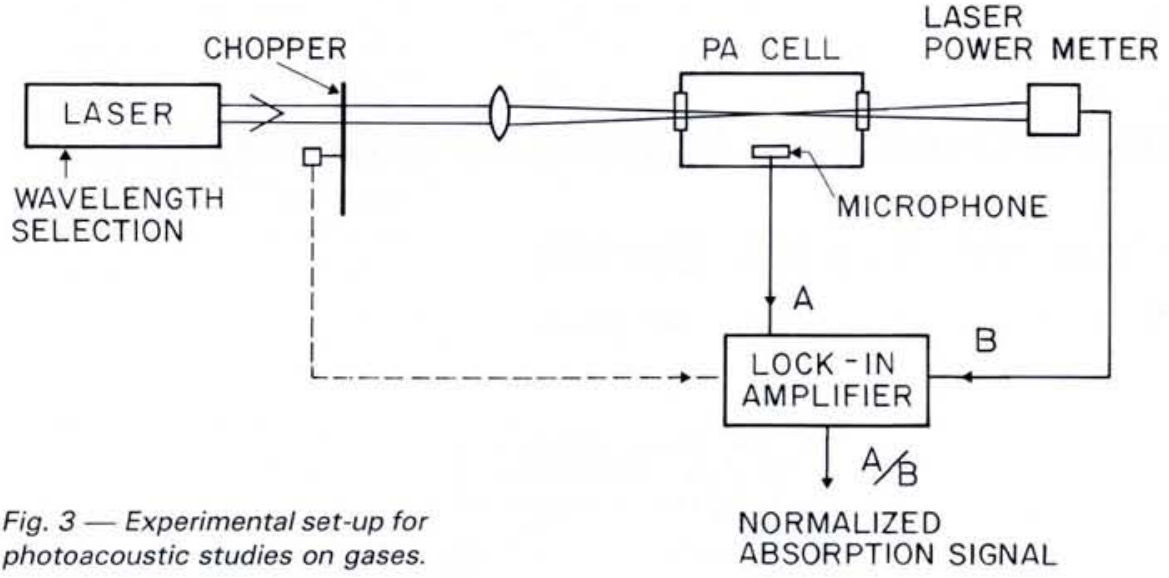

face reflectivity or slight deformation of the surface (photothermal displacement) can also be detected without contact by a probe beam. Finally, variations of the thermal radiation from the surface can be monitored with an infrared detector (photothermal radiometry). Other techniques include pyroelectric detection in thin films, thermal lensing and interferometric methods.

Studies on liquids are usually performed with pulsed lasers and piezoelectric or probe beam deflection monitoring of the generated acoustic signal. The most widely used arrangement for gases employs a chopped laser beam directed through an appropriate gas cell where the pressure modulations are detected either by commercial condenser microphones with sensitivities up to $100 \mathrm{mV} / \mathrm{Pa}$ or by miniature electret microphones with sensitivities of typically $10 \mathrm{mV} / \mathrm{Pa}$. These acoustic detectors are easy to use and their response is appropriate for most PA studies. The schematic set-up is shown in Fig. 3 . The phasesensitive detection of the microphone signals by the lock-in amplifier suppresses any broadband background noise. The transmitted laser power, indicated by $B$, is used for normalizing the $P A$ signal so that the $B / A$ signal is directly proportional to the absorption coefficient of the sample at the laser wavelength.

\section{Applications of PAS}

Photoacoustic and photothermal phenomena have been widely used for numerous non-spectroscopic applications like the determination of thermal diffusivity, non-destructive testing of materials (in particular the probing of sub-surface defects) by thermal wave imaging, monitoring de-excitation processes, studies of phase transitions, etc. Here only the spectroscopic applications are considered as they demonstrate the main characteristics and the potential of PA spectroscopy.

\section{Studies on Liquids}

In principle there are five important interaction mechanisms which can be responsible for the excitation of acoustic waves in liquids by the impact of pulsed laser radiation: dielectric break down, vaporization, thermoelasticity, electrostriction and radiation pressure. Their contributions depend on the parameters of the incident laser beam as well as on the optical and thermal properties of the medium. PAS studies on liquids are essentially based on the pure thermoelastic effect where the thermal expansion of the laser-heated volume generates the acoustic wave. Experimental and theoretical studies have been performed for a wide absorption range including "transparent" and opaque liquids.

In the first case, minimum absorption coefficients of $10^{-6} \mathrm{~cm}^{-1}$ have been detected corresponding to absorbed laser pulse energies of only $10^{-9} \mathrm{~J}$. Owing to the high sensitivity, the method has been successfully applied e.g. to the measurement of the 8th harmonic of the $\mathrm{C}-\mathrm{H}$ fundamental stretching vibration of benzene and to the detection of impurities in transparent liquids.

Depending on the penetration depth and the actual laser beam diameter, plane or hemispherical sound waves are excited in opaque liquids for most media within the IR wavelength range. In this case, different boundaries, i.e. surface conditions as e.g. free and confined surfaces or optically thin and thick surface films, can be investigated. PAS allows us to analyze and monitor surface films on liquids in situ in a non-contact and rather simple manner.

A recent example concerns the monitoring of polymerization in a surface film. The sample consisted of an optically thick layer $(100 \mu \mathrm{m})$ of octadecyl methacrylate (ODMA, $\mathrm{C}_{22} \quad \mathrm{H}_{42} \mathrm{O}_{2}$ ) floating on a water surface. This layer can be polymerized in a nitrogen atmosphere by UV irradiation at room temperature. During the polymerization process the absorption band of ODMA centred at $940 \mathrm{~cm}^{-1}$ diminishes and finally vanishes completely. We used this fact for PA monitoring of the polymerization by measuring the PA signals which are excited by the impact of $\mathrm{CO}_{2}$ laser pulses at different wavelengths as a function of the UV irradiation time

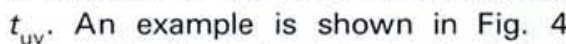
where the time dependence is plotted for the $10 P(28)$ laser transition located at the centre of the ODMA absorption band and the $10 P(20)$ transition situated on the edge of the band. At the time $t_{\mathrm{uv}}=0$, i.e. before polymerization 


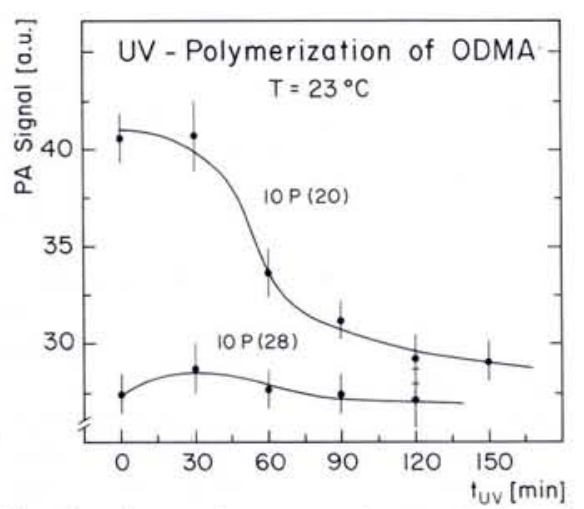

Fig. 4 - Laser-photoacoustic monitoring of photopolymerization of ODMA layer. Measured amplitudes vs UV irradiation time $t_{u v}$.

starts, the PA amplitude is considerably larger for the $10 P(20)$ than for the $10 \mathrm{P}(28)$ laser transition.

\section{Studies on Gases}

Numerous laser-PA studies have been performed in gaseous media including studies on the absorption by molecules in excited states, and investigations on chemically reactive gases which may produce transient intermediate chemical species. Most applications, however, concern trace gas monitoring. In addition to common PAS, various spectroscopic schemes with PA detection have been applied. These include Doppler-free spectroscopy, vibrational overtone spectroscopy, PA Raman spectroscopy, harmonic saturated spectroscopy as well as non-linear spectroscopy, i.e. two-photon and infrared multiphoton absorption.

Today, atmospheric pollution by numerous trace constituents at $\mathrm{ppmV}$ $\left(10^{-6}\right)$ or ppbV $\left(10^{-9}\right)$ concentrations is of major concern. Different techniques have been introduced for the detection of trace gases which is a prerequisite for understanding the complex chemistry of the atmosphere, particularly photochemical smog formation. However, since conventional methods like chemiluminescence, common IR spectroscopy, flame ionization, gas chromatography etc. do not meet all the requirements, novel schemes have to be studied for the continuous, sensitive and selective monitoring of numerous pollutants either in situ or remotely.

For in situ applications PAS can be characterized by the following features: (i) High sensitivity which permits trace gas detection at the ppbV-level under interference-free conditions.

(ii) Simple set-up, e.g. no cryogenic cooling for a radiation detector is required. (iii) Easy calibration with certified gas mixtures.

(iv) Wide dynamic range, i.e. linear response over several orders of magnitude with respect to concentration. The method is thus suitable for both immission and emission studies.

(v) Detection of numerous components with one single apparatus, equipped e.g. with a tunable IR laser, is possible.

(vi) Only a short absorption path is required, i.e. wavelengths beyond atmospheric windows can be applied.

(vii) Continuous in situ measurements on flowing air with suitable PA cells are possible.

Since most molecules exhibit characteristic absorption features within the mid IR range, the so-called fingerprint region, tunable lasers emitting in this wavelength range are most appropriate for trace gas detection. In the course of our own studies in this field we have built two computer-controlled PA systems: a stationary apparatus with a $\mathrm{CO}$ laser and a mobile system with a $\mathrm{CO}_{2}$ laser. Both lasers are only line-tunable, not continuous, which poses special requirements on the analysis of measured PA spectra of multicomponent gas mixtures with interfering absorptions. The analysis is performed on the basis of calibration spectra of single trace constituents by using a new iterative formalism. This procedure permits the determination of individual concentrations as well as the evaluation of the best laser transitions for the detection of specific components, yielding a minimum of interference with other components at optimum sensitivity.

A specific application concerns the analysis of motor vehicle exhaust gases performed with the CO laser PA system. Since water vapour exhibits a considerable absorption in the wavelength range between $5.15 \mu \mathrm{m}$ and $6.35 \mu \mathrm{m}$ of our $\mathrm{CO}$ laser, we have used a dual-beam arrangement with specially designed sample and reference PA cells. In spite of the water-vapour interference we have been able to derive the concentrations of 12 of the most important components like nitric oxide, ethylene, propylene, aromatic hydrocarbons and aldehydes at ppmV and sub-ppmV con- centrations. It should be emphasized that even the isomers of xylene can be determined separately. This separation is difficult, if not impossible, to achieve with conventional methods, e.g. with gas chromatography.

Our second PA apparatus is equipped with a sealed-off $\mathrm{CO}_{2}$ laser, line-tunable between $9.2 \mu \mathrm{m}$ and $10.8 \mu \mathrm{m}$. A special design of the acoustically resonant PA chamber permits continuous measurements on air samples with a flow rate of ca $1 \mathrm{l} / \mathrm{min}$ without loss in sensitivity. The minimum detectable absorption coefficient is $10^{-8} \mathrm{~cm}^{-1}$ which corresponds to trace gas concentrations of $\mathrm{ppbV}\left(\mu \mathrm{g} / \mathrm{m}^{3}\right)$ under interference-free conditions. For the in situ monitoring of air pollutants, the entire PA apparatus is installed in a mobile airconditioned trailer of $4 \mathrm{~m}$ length.

Hitherto, our mobile system has been applied at various locations for the in situ recording of specific pollutants like ethylene $\left(\mathrm{C}_{2} \mathrm{H}_{4}\right)$ in outdoor air. Since today considerable amounts of $\mathrm{C}_{2} \mathrm{H}_{4}$ are emitted in the atmosphere by road traffic and various industries, the enhanced $\mathrm{C}_{2} \mathrm{H}_{4}$ concentrations are a continual source of stress for plant growth in urban areas. Furthermore, $\mathrm{C}_{2} \mathrm{H}_{4}$ acts as a precursor substance for photochemical smog. The $1 \mathrm{OP}(14) \mathrm{CO}_{2}$ laser transition at $949.49 \mathrm{~cm}^{-1}$ is ideally suited for detecting $\mathrm{C}_{2} \mathrm{H}_{4}$ because of the proximity of the $\mathrm{C}_{2} \mathrm{H}_{4}$ vibrational band $v_{7}$ located at $948.77 \mathrm{~cm}^{-1}$. With the resulting large absorption cross section of $1.3 \times 10^{-18} \mathrm{~cm}^{2}$ we achieve a detection limit for $\mathrm{C}_{2} \mathrm{H}_{4}$ in ambient air of below $5 \mathrm{ppbV}$ if attention is paid to the interfering absorption by $\mathrm{CO}_{2}$. Diurnal concentration profiles are correlated with the strength of traffic, wind data and, on sunny days, with the local ozone production.

Recent studies which demonstrate the versatility of PAS concern in situ measurements on industrial exhaust air which represents a mixture of numerous components with concentrations varying in time. The continuous record-
Fig. 5 - Concentration profile of ethanol-vapour in industrial exhaust air.

$\begin{aligned} & \longrightarrow \text { : Laser-photoacoustic } \\ & \text { monitoring, } \\ & \times \quad \text { : Gas chromatography }\end{aligned}$ data.

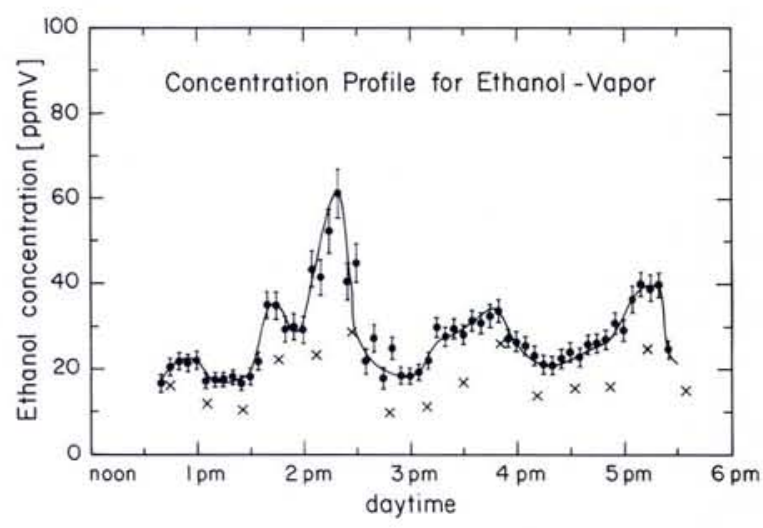


ing of the main constituents is thus of great importance. As an example Fig. 5 shows the PA monitoring of ethanol $\left(\mathrm{C}_{2} \mathrm{H}_{5} \mathrm{OH}\right)$-vapour present in the exhaust stream of a pharmaceutical production plant besides numerous other volatile organic compounds. The PA measurements were performed periodically at selected $\mathrm{CO}_{2}$ laser transitions yielding ethanol and methanol concentrations simultaneously. In general, good agreement between the photoacoustically derived concentrations and independent gaschromatographic (GC) measurements (represented by $\mathrm{x}$ in Fig. 5 ) is obtained under steady state conditions. The PA data are not systematically higher than the GC data as could be supposed from the results in Fig. 5 , but the time resolution offered by PAS is better and can be exploited for the monitoring of even short-term concentration fluctuations.

\section{Perspectives}

Since photoacoustics involves optical, thermal and acoustical phenomena, photoacoustic and photothermal sensing schemes are ideally suited for studying various material properties. Apart from non-spectroscopic applications like e.g. thermal wave imaging which today represents an important technique for the non-destructive evaluation (NDE) of materials, laser-PA spectroscopy offers great potential for applications ranging from surface physics and chemistry to environmental sciences. The few examples discussed indicate the wide gamut of PAS applications that can be expected.

\section{Acknowledgement}

The author is grateful to S. Bernegger and P.L. Meyer for their collaboration. Our photoacoustic studies were funded by the Swiss National Science Foundation (National Research Program 14) and ETH Zurich.

\section{FURTHER READING}

Zharov V.P. and Letokhov V.S., Laser Optoacoustic Spectroscopy, Springer Ser. Opt. Sciences, Vol. 37 (Springer Berlin, Heidelberg) 1986.

Tam A.C., Rev. Mod. Phys. 58 (1986) 381. 431.

Sigrist M.W., J. Appl. Phys. 60 (1986) R83R121.

Rothberg L., J. Phys. Chem. 91 (1987) 3467-3474.

Photoacoustic and Photothermal Phenomena, Eds. P. Hess and J. Pelzl, Springer Ser. Opt. Sciences, Vol. 58 (Springer Berlin, Heidelberg) 1988.

Sigrist M.W., Bernegger S. and Meyer P.L., Infrared Phys. 29 (1989) 805-814.

Photoacoustic, Photothermal and Photochemical Processes in Gases, Ed. P. Hess, Top. Curr. Phys., Vol. 46 (Springer Berlin, Heidelberg) 1989.

Photoacoustic, Photothermal and Photochemical Processes at Surfaces and in Thin Films, Ed. P. Hess, Top. Curr. Phys. Vol. 47 (Springer Berlin, Heidelberg) 1989.

\section{EQEC: 2nd European Quantum Electronics Conference}

\section{Dresden, 28 August - 1 September 1989}

Following EQEC-1, the first Divisional Conference of the Quantum Electronics Division held in Hannover in 1988, EQEC-2 took place in Dresden's Town Hall under the auspices of the GDR Academy of Sciences, the Friedrich Schiller University, Jena, and the Physical Society of the GDR.

The four plenary papers highlighted present mainstreams in quantum electronics. Akhmanov (Moscow) reported on non-linear optics in 2-dimensional systems, discussing non-linear wave dynamics, large scale transverse wave interaction, optical turbulence and spatial squeezing. These abstract concepts were beautifully illustrated by experimental results shown in an impressive movie. Welling (Hannover) reviewed the many routes towards very-short-wavelength lasers and their potential applications. Glauber (Cambridge) discussed the theory of radiating atoms in resonant cavities, while Paul (Berlin) addressed a basic issue in quantum optics, namely interference between independent single photons, touching upon basic issues in quantum measurement theory.

An important contribution in the area of laser physics was made by Bimberg (W. Berlin) who reported on gain switching of semiconductor lasers where picosecond pulses can be produced by driving the laser with a much longer electrical pulse in such a way that only the first relaxation oscillation spike occurs. Dunn (St. Andrews) discussed the use of excimer lasers to pump optical parametric oscillators (OPO's) in the visible. Generally speaking OPO's have been of marginal importance during the past 25 years but they are now rapidly gaining popularity owing to new nonlinear materials and new pump sources becoming available. Prior (Rehovot) addressed current models used to describe laser fluctuations, namely phase diffusion, random phase jumps and telegraph phase noise, generalizing these into a non-Markovian stochastic jump model. Distributed feedback gas lasers were analysed in their own right, and as a model system for DFB solid state lasers, by Kneubühl (Zurich). DFB gas lasers allow easy tests of theory since their parameters are much better controlled than in the case of a DFB semiconductor laser. Lasers were also addressed by Ovaevski (Moscow) in the context of the intriguing analogy between lasing action and superconductivity that can be made by comparing Cooper pairs with photons.

Regarding non-linear optical materials, Kobayashi (Tokyo) considered the problems which arise when one has to assess the many new materials (particularly polymers) which are presently being developed for fast optical switches and optical logic. He introduced a simple method based on measuring the transmission spectrum of a polymer layer sandwiched between two transparent electrodes. Stockman (Novosibirsk) gave a very interesting presentation of the giant optical non-linearities of fractal clusters such as aggregates of colloidal gold.

Walther (Max-Planck-Institut, Garching) reported on recent single-atom experiments which allow many early "Gedanken" experiments to be performed in the laboratory. Such experiments may resolve some basic issues in quantum physics since ensemble averaging is no longer present. Another significant contribution in a related area included the report by Chebotaev (Novosibirsk) on synchronisation in quantum transitions, making connections with Ramsey fringes, photon echoes and free induction decay. Possible applications would be super high resolution spectroscopy and high-speed atomic memory systems. In a theoretical paper Stenholm (Helsinki) touched upon the issue of Landau-Zener crossing during a laser assisted collision. For reasons not yet understood the range of validity of the "classical" Landau-Zener result is apparently much larger than expected. Finally, Noordam (Amsterdam) reported recent results obtained in producing electronic wave packets in Rydberg atoms using picosecond laser excitation. A full set of abstracts of all invited and contributed papers has been published in Volume 13D of the Europhysics Conference Abstracts series which is available from the EPS Secretariat.

J.P. Woerdman, Leiden 